\title{
Dependence of a planet's chaotic zone on particle eccentricity: the shape of debris disc inner edges
}

\author{
Alexander J. Mustill ${ }^{1,2 \star}$ and Mark C. Wyatt ${ }^{2}$ \\ ${ }^{1}$ Departamento de Física Teórica, Universidad Autónoma de Madrid, Cantoblanco, 28049 Madrid, Spain \\ ${ }^{2}$ Institute of Astronomy, University of Cambridge, Madingley Road, Cambridge CB3 OHA
}

Accepted 2011 October 4. Received 2011 October 3; in original form 2011 August 8

\begin{abstract}
The orbit of a planet is surrounded by a chaotic zone wherein nearby particles' orbits are chaotic and unstable. It was shown by Wisdom that the chaos is driven by the overlapping of mean motion resonances which occurs within a distance $(\delta a / a)_{\text {chaos }} \approx 1.3 \mu^{2 / 7}$ of the planet's orbit. However, the width of mean motion resonances grows with the particles' eccentricity, which will increase the width of the chaotic zone at higher eccentricities. Here we investigate the width of the chaotic zone using the iterated encounter map and $N$-body integrations. We find that the classical prescription of Wisdom works well for particles on low-eccentricity orbits. However, above a critical eccentricity, dependent upon the mass of the planet, the width of the chaotic zone increases with eccentricity. An extension of Wisdom's analytical arguments then shows that, above the critical eccentricity, the chaotic zone width is given by $(\delta a / a)_{\text {chaos }} \approx$ $1.8 e^{1 / 5} \mu^{1 / 5}$, which agrees well with the encounter map results. The critical eccentricity is given by $e_{\text {crit }} \approx 0.21 \mu^{3 / 7}$. This extended chaotic zone results in a larger cleared region when a planet sculpts the inner edge of a debris disc composed of eccentric planetesimals. Hence, the planet mass estimated from the classical chaotic zone may be erroneous. We apply this result to the HR 8799 system, showing that the mass of HR $8799 \mathrm{~b}$ inferred from the truncation of the disc may vary by up to 50 per cent depending on the disc particles' eccentricities. With a disc edge at $90 \mathrm{au}$, the necessary mass of planet $\mathrm{b}$ to cause the truncation is 8-10 Jovian masses if the disc particles have low eccentricities $(\lesssim 0.02)$, but only 4-8 Jovian masses if the disc particles have higher eccentricities. Our result also has implications for the ability of a planet to feed material into an inner system, a process which may explain metal pollution in white dwarf atmospheres.
\end{abstract}

Key words: chaos - celestial mechanics - planets and satellites: dynamical evolution and stability - circumstellar matter - stars: individual: HR 8799.

\section{INTRODUCTION}

Planets strongly affect the dynamics of bodies on nearby orbits. Bodies coming within around one Hill radius are strongly perturbed on the synodic time-scale and can either be scattered, resulting eventually in collision with the star or ejection from the system, or collide with the planet. However, beyond this region there is a chaotic zone of unstable orbits. Here, orbits diffuse through phase space and may eventually find themselves intersecting the planet's orbit, which will again result in a strong scattering or collision event. This unstable chaotic zone is thought to be responsible for sculpting the inner edges of debris discs such as that around Fomalhaut (Quillen 2006a; Kalas et al. 2008; Chiang et al. 2009), with the location and

^E-mail: alex.mustill@uam.es shape of the disc edge being determined by the mass and location of the planet. The zone may also provide a reservoir for feeding bodies from an outer disc into the inner system. This is likely to be important in the late stages of stellar evolution, when scattering of planetesimals by planets has been invoked to explain metal pollution observed in some white dwarf atmospheres as well as hot discs around them (Zuckerman et al. 2003; Farihi, Jura \& Zuckerman 2009; Bonsor, Mustill \& Wyatt 2011).

Chaos in dynamical systems is often driven by overlapping resonances (Chirikov 1979). Wisdom (1980) derived an analytical formula for the extent of a planet's chaotic zone based on overlapping first-order mean motion resonances, finding

$(\delta a / a)_{\text {chaos }}=c \mu^{2 / 7}$,

where $\mu=m_{\mathrm{pl}} / m_{\star}$ is the ratio of planetary to stellar mass and the coefficient $c=1.3$. This has been confirmed numerically, both using 
iterations of the encounter map (Duncan, Quinn \& Tremaine 1989; see Section 2 below) and in full $N$-body integrations (Chiang et al. 2009), although the coefficient $c$ when determined numerically is somewhat higher. However, the result of Wisdom (1980) is only valid for low-eccentricity particles, since the widths of mean motion resonances increase at larger eccentricities (Murray \& Dermott 1999). Quillen \& Faber (2006) extended Wisdom's result to consider the behaviour of particles orbiting close to an eccentric planet with low free eccentricities, which is relevant for modelling the Fomalhaut disc. They showed that the width of the zone for particles orbiting close to an eccentric planet with low free eccentricities is the same as that for low-eccentricity particles orbiting close to a circular planet.

The effect the particles' free eccentricities have on the width of the chaotic zone has not been well explored. Since the Kuiper belt objects of our own Solar system can have free eccentricities of 0.1 and above, one might expect the debris discs of some extra-Solar systems to be stirred to a similar degree. Bonsor et al. (2011) found, in $N$-body integrations, that the width of the chaotic zone for more eccentric particles ( $e$ up to 0.1 ) appeared to be greater than that for less eccentric particles. The small number of particles in the $N$ body integrations did not permit definite conclusions to be drawn, but they confirmed the increasing width using the computationally more efficient iterated encounter map (Duncan et al. 1989). Bonsor et al. (2011) speculated that the increasing width of the zone was due to the particles' pericentres entering the chaotic zone. This, however, seems unlikely since the chaos is caused by resonance overlap and that depends on the particles' semimajor axes and not directly on their pericentres. Here we demonstrate that the increasing chaotic zone width is instead due to the increasing width of mean motion resonances as particles' eccentricities are increased. Quillen (2007) and Chiang et al. (2009) investigated the effects of non-gravitational effects such as collisions and radiation forces on the inner edge of the disc, and here we investigate the effects of the particles' eccentricity.

One reason why it is important to characterize this chaotic zone properly is that currently much interest revolves around the effects that perturbing planets can have on debris disc morphology, particularly with a view to determining or refining the parameters of known or suspected planets. Accordingly, the $\mu^{2 / 7}$ law has been used to constrain the mass and semimajor axis of planets that may be sculpting the inner edges of debris discs (e.g. Moerchen et al. 2011), to verify the consistency of planet and disc parameters determined through other means (e.g. Su et al. 2009), and to aid setting up and interpreting $N$-body integrations (e.g. Chiang et al. 2009) studying the sculpting of debris disc edges. Hence, refinements to and deviations from this law are of prime importance for understanding the interaction of planets and debris discs. Given the large uncertainties which attend determinations of masses of planets detected by direct imaging (Kalas et al. 2008), dynamical constraints are very valuable and use of accurate dynamical models should be made. In Section 4.2 of this paper, we discuss the HR 8799 system and how mass estimates of planet $b$, thought to be sculpting the inner edge of the cold debris disc, are affected by deviations from the $\mu^{2 / 7}$ law.

This paper is organized as follows. In Section 2, we use the encounter map and $N$-body integrations to explore the chaotic zone as a function of particle eccentricity. In Section 3, we derive the width of the chaotic zone for eccentric particles using a resonance overlap criterion. In Section 4, we discuss the implications of this for studies of debris discs sculpted by planets, particularly the HR 8799 system, and planetary systems orbiting white dwarfs.

\section{NUMERICAL INVESTIGATION}

We investigated the structure of the chaotic zone using the iterated encounter map described in Duncan et al. (1989). This uses the approximate solution to Hill's equations calculated by Henon \& Petit (1986), which assumes a moderate separation between the planet and particle, to calculate the impulsive change to a particle's orbital elements due to a conjunction with the planet every synodic period. We determined whether orbits were regular or chaotic by examining the Fourier transform of the eccentricity evolution. Regular orbits have power spectra with a few well-defined peaks, while the more chaotic an orbit the more peaks its power spectrum displays (this is a consequence of the phase space of a chaotic dynamical system being densely filled with periodic orbits). We chose a critical number of peaks, a peak being defined as where the slope of the power spectrum changes from positive to negative, to decide between regular and chaotic trajectories. For the investigations below, with $10^{4}$ iterations, we found 1000 peaks distinguished regular and chaotic orbits over the whole range of parameters considered. This classification method is similar to the spectral number chaos indicator (Zhou, Dvorak \& Sun 2011).

In this way, we then investigated the nature of trajectories as a function of the particles' semimajor axis $\epsilon=\left(a-a_{\mathrm{pl}}\right) / a_{\mathrm{pl}}$ and eccentricity $e$, and the planet:star mass ratio $\mu$. The results are shown in Fig. 1. In these plots, each point in the grid represents 100 particles, with initially random values of longitude of pericentre and mean anomaly. The grey-scale shows the fraction of particles whose orbits were classed as chaotic, with black representing 100 per cent regular and white 100 per cent chaotic. In the region at the top left corner above the black line, the particles are on planet-crossing orbits and hence the encounter map is invalid. These particles were classed as chaotic since they will be unstable anyway, except perhaps for a few protected from close encounters by resonances.

From Fig. 1 we see that the edge of the chaotic zone is a function of eccentricity, with the more eccentric particles being chaotic at greater separations from the planet than their less eccentric brethren. There are two regimes: a low-eccentricity regime in which the chaotic zone is independent of eccentricity, and a moderate-eccentricity regime where the width of the chaotic zone increases with eccentricity. The former regime is that identified by Wisdom (1980). The critical eccentricity separating these regimes increases with $\mu$. The width at low eccentricity is in good agreement with the result from the resonance overlap criterion (Wisdom 1980; shown by a vertical blue line), although this systematically underestimates it, as previous studies have shown (Duncan et al. 1989). The increased chaotic zone width at larger eccentricities is likely due to the increasing width of the mean motion resonances at higher eccentricities, which can clearly be seen as intrusions of chaotic motion into the regions of regular motion. This increasing width can be derived analytically, as shown below.

To verify the accuracy of the encounter map results, we ran an $N$-body integration using the hybrid algorithm included in the MERCURY package (Chambers 1999). 961 test particles were placed on orbits exterior to a planet of mass $\mu=10^{-8}$ and their orbits integrated for $10 \mathrm{Myr}$. These parameters correspond to panel (f) of Fig. 1. Their final eccentricities and semimajor axes are shown in Fig. 2. Initially, they fill the shaded region, but many are removed from regions closer to the planet and scattered on to orbits which almost intersect the planet's (shown by the dashed black line). The vertical solid blue line shows the chaotic zone width according to Wisdom (1980). More eccentric particles are destabilized at greater 
(a) $\mu=10^{-3}$

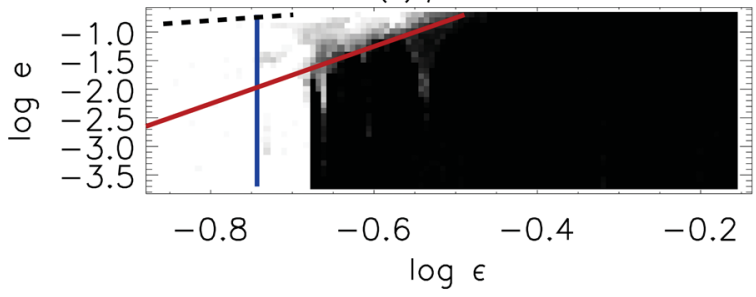

(c) $\mu=10^{-5}$

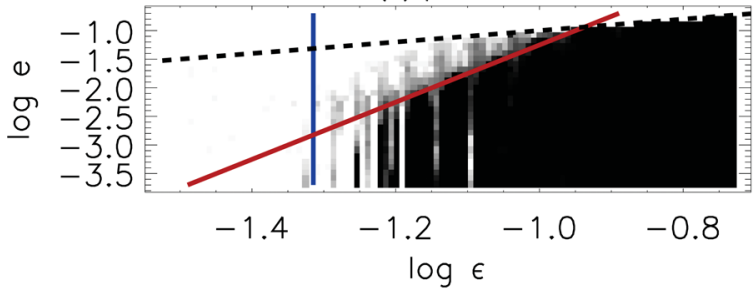

(e) $\mu=10^{-7}$

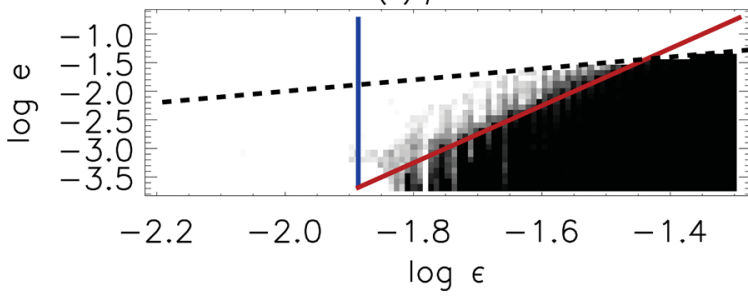

(b) $\mu=10^{-4}$

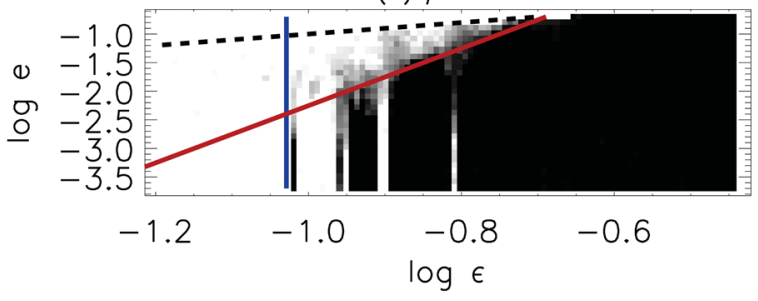

(d) $\mu=10^{-6}$

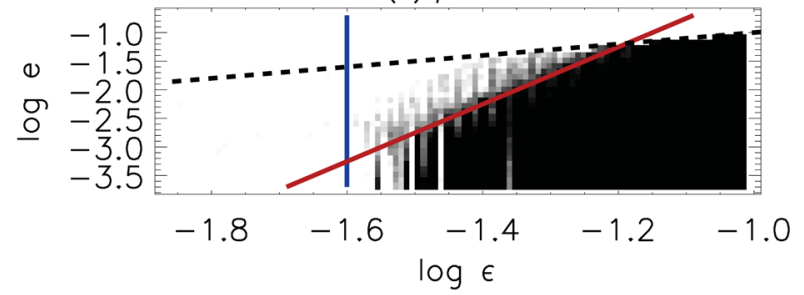

(f) $\mu=10^{-8}$

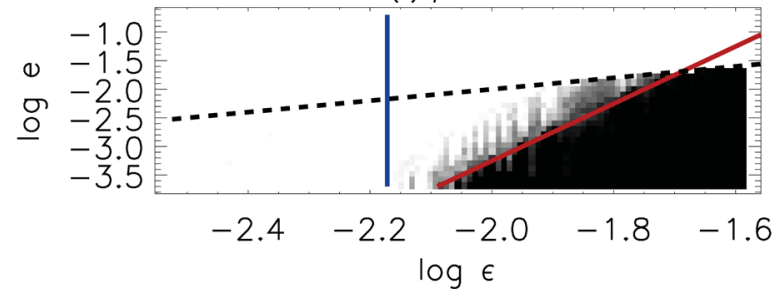

Figure 1. (a)-(f): extent of chaotic zone as a function of particle eccentricity and semimajor axis and planet mass. The right-hand side of each plot is at two Hill radii from the planet. The vertical blue line shows the extent of the classical chaotic zone (Wisdom 1980). Above the dashed black line, the particles are on planet-crossing orbits; these particles were all classed as chaotic regardless of the map behaviour since they will experience close encounters with the planet. The solid red line shows the analytical estimate of the chaotic zone width as a function of eccentricity (equation 10).

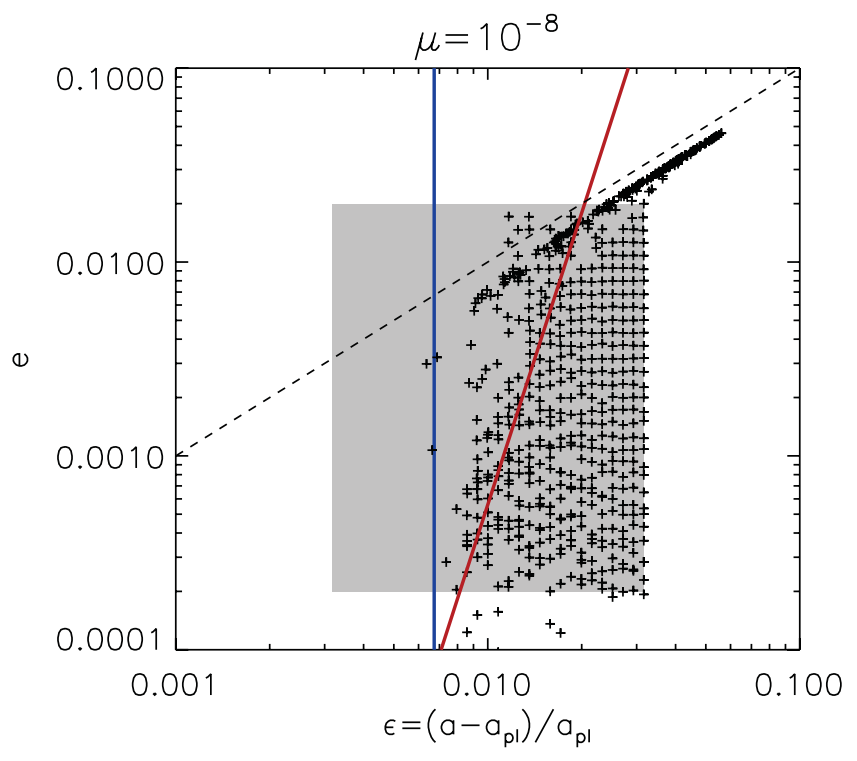

Figure 2. Fates of 961 particles after a $10 \mathrm{Myr} N$-body integration. Initially, they are distributed over the grey rectangle and their final semimajor axes and eccentricities are plotted as crosses. Also shown are the same lines as in Fig. 1: dashed black - planet-crossing orbits; solid blue - classical chaotic zone; solid red - extended chaotic zone. Dynamical clearing above the red line is apparent. semimajor axes than less eccentric ones, as was seen in the results from the encounter map. Hence, the encounter map appears to be giving an accurate picture of the dynamics. Additionally, the region above the dashed black line is almost totally devoid of particles, due to scattering by the planet (these particles either have collided with the planet or are in the unstable population just below the black line which is in the process of being scattered). This justifies our labelling of planet-crossing orbits as 'chaotic' in Fig. 1.

\section{ANALYTICAL DERIVATION}

The standard derivation of the chaotic zone width (Wisdom 1980; Quillen \& Faber 2006) takes the widths of the mean motion resonances to be independent of eccentricity. Working from the resonance Hamiltonian,

$\mathcal{H}=J^{2}+\beta J-J^{1 / 2} \cos \theta$,

where $J \propto e^{2}$ is the canonical momentum, $\theta$ is the resonant argument, and $\beta$ is a parameter measuring distance to the nominal resonance location (see Quillen 2006b; Mustill \& Wyatt 2011), one can show that the width of a first-order resonance at low eccentricity is approximately (Wisdom 1980)

$(\delta a / a)_{\mathrm{res}}=3.2 \mu^{2 / 3} j^{1 / 3}$,

where $j$ numbers the resonance, $j:(j-1)$. In the limit of large $j$, adjacent resonances are separated by a distance

$(\delta a / a)_{\mathrm{sep}}=2 /\left(3 j^{2}\right)$. 


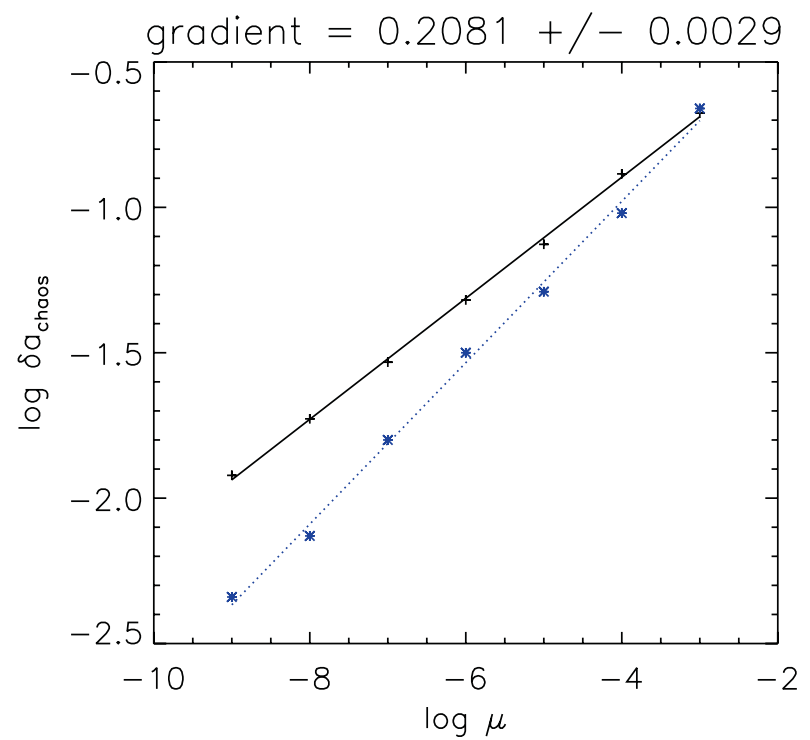

Figure 3. Black, solid line and crosses: the chaotic zone width at a moderate eccentricity of $e=0.01$ as a function of planet:star mass ratio. Points show the numerical results from the grids in Fig. 1, and the line shows the regression line, given by $(\delta a / a)_{\text {chaos }}=0.86 \mu^{0.208}$, close to the analytical result predicted by equation (10). Blue, dotted line and stars: the chaotic zone width at zero eccentricity. Here the regression line (dotted) has a slope of $0.278 \pm 0.0077$, close to the $2 / 7$ predicted by the analytical arguments of Wisdom (1980).

Equating these two distances gives the outermost resonance where overlap occurs as

$j=0.51 \mu^{-2 / 7}$.

Since $a-a_{\mathrm{pl}}=2 /(3 j)$ for large $j$, the chaotic zone width is thus given by

$(\delta a / a)_{\text {chaos }}=1.3 \mu^{2 / 7}$.

This scaling is successfully reproduced by numerical iterations of the encounter map (Duncan et al. 1989, and Fig. 3 of this paper).

However, the resonance width grows with particle eccentricity, as can be seen in panel (a) of Fig. 1. This means that resonances that do not overlap at low eccentricities may overlap at higher eccentricities, and cause the chaotic zone to be wider. When eccentricities are not small, the width of the resonance can be found by considering the width of the resonant libration region after the resonant separatrix forms, which is given by $\delta J=4(4 J)^{1 / 4}$ (Murray \& Dermott 1999) in terms of the canonical momentum $J$. Now $J$ is related to $e$ by

$J=k_{j}\left(m_{\mathrm{pl}} / \mathrm{M}_{\oplus}\right)^{-2 / 3}\left(m_{\star} / \mathrm{M}_{\odot}\right)^{2 / 3} e^{2}$,

where $k_{j} \sim\left(3 j \mathrm{M}_{\odot} / 2 \mathrm{M}_{\oplus}\right)^{2 / 3} / 2$ in the limit of large $j$ (Quillen \& Faber 2006; Mustill \& Wyatt 2011), and so

$\delta J=1.3 \mu^{-2 / 3} j^{2 / 3} e \delta e$.

Using the relationship between eccentricity change and semimajor axis change in a resonance $\delta a=2 j a e \delta e$ (Murray \& Dermott 1999), we then have

$(\delta a / a)_{\mathrm{res}}=7.78 e^{1 / 2} \mu^{1 / 2} j^{1 / 2}$,

for the maximum libration width in semimajor axis. Equating this to the resonance separation and redoing the previous derivation then give

$(\delta a / a)_{\text {chaos }}=1.8 e^{1 / 5} \mu^{1 / 5}$ as the width of the chaotic zone at higher eccentricities. Note the two implications of this equation. First, the chaotic zone width grows weakly with eccentricity. The dependence is shown in Fig. 1 as solid red lines. The agreement with the results from the encounter map is striking. We also show the dependence in Fig. 2, where we can see many unstable particles above the solid red line. Secondly, the $\mu^{2 / 7}$ dependence of the chaotic zone width on planet mass, valid in the low-eccentricity regime, is replaced with a $\mu^{1 / 5}$ dependence. To verify this, in Fig. 3 we plot the width of the chaotic zone at an eccentricity of $e=0.01$ as a function of $\mu$. The width was determined by finding the innermost cell where all trajectories were regular from the grids presented in Fig. 1. The regression line through these points gives a dependence $(\delta a / a)_{\text {chaos }}=0.86 \mu^{0.208}$, in excellent agreement with the analytical result from equation (10). Thus, for higher eccentricities, the chaotic zone width is given by a $\mu^{1 / 5}$ scaling, not the more familiar $\mu^{2 / 7}$. For comparison, the width of the chaotic zone at $e=0$ as a function of $\mu$ is shown in the same figure. The regression line here gives $(\delta a / a)_{\text {chaos }}=1.35 \mu^{0.278}$, in excellent agreement with Wisdom's original result.

Equation (10) also allows the critical eccentricity separating the two regimes to be estimated. Equating $(\delta a / a)_{\text {chaos }}$ obtained from equation (10), for eccentric particles, with the value from equation (6), for low-eccentricity particles, gives

$e_{\text {crit }} \approx 0.21 \mu^{3 / 7}$,

growing with $\mu$, as is seen in Fig. 1. Note that $e=0.01$ is the critical eccentricity for $\mu=10^{-3}$, and indeed the widths of the classical and extended zones coincide at this point (Fig. 3).

Note that there is a maximum eccentricity for which this derivation of the extended chaotic zone is valid. This occurs where the solid red line in Fig. 1 marking the boundary of the extended chaotic zone intersects the dashed black line denoting the region of planet-crossing orbits. At eccentricities above this, particles will be removed directly by close encounters rather than via chaotic diffusion. The maximum eccentricity is given by

$e_{\max }=2.1 \mu^{1 / 4}$,

and in this regime particles are unstable if

$\delta a / a<e$.

In the next section, we adopt the slightly more conservative condition that particles are also unstable if they approach within one Hill radius of the planet.

\section{DISCUSSION}

\subsection{The shape of debris disc inner edges}

This result has important implications for the interactions of planets and debris discs. Discs showing inner clearings are commonly explained by the existence of a planet removing particles from its chaotic zone (e.g. Quillen 2006a). The mass and semimajor axis of the planet determine both the location and the sharpness of the inner edge (Quillen 2006a; Quillen \& Faber 2006; Chiang et al. 2009). Since our investigations above have revealed that the extent of the chaotic zone differs from the classical prescription, we now explore its effect on the nature of inner edges to debris discs. As discussed by Chiang et al. (2009), to correctly model the shape of a disc edge it is necessary to account not only for particles located in semimajor axis at the edge of the chaotic zone, but also ones further out whose eccentricities may cause them to affect the radial distribution of particles at the disc edge. Accordingly, we generate surface 
density profiles for discs in the following way. We consider simple model discs where $5 \times 10^{4}$ planetesimals are uniformly distributed in eccentricity between 0 and a maximum $e_{\mathrm{m}}$ and semimajor axis between 1 and 2 au. Particles are removed if their orbits under iterations of the encounter map are chaotic, or if their periapsis comes within one Hill radius of the planet. We then sample 100 mean anomalies from each particle's orbit, and sum the particles in radial bins to find the surface density profile. We also vary the particles' eccentricities and semimajor axes according to the encounter map results, because the particles do not remain at their initial $a$ and $e$ even if their orbits are regular.

The results, for mass ratios $\mu=10^{-3}, 10^{-4}$ and $10^{-5}$ and a maximum eccentricity of $e_{\mathrm{m}}=0.1$, are shown in the top panel of Fig. 4 as
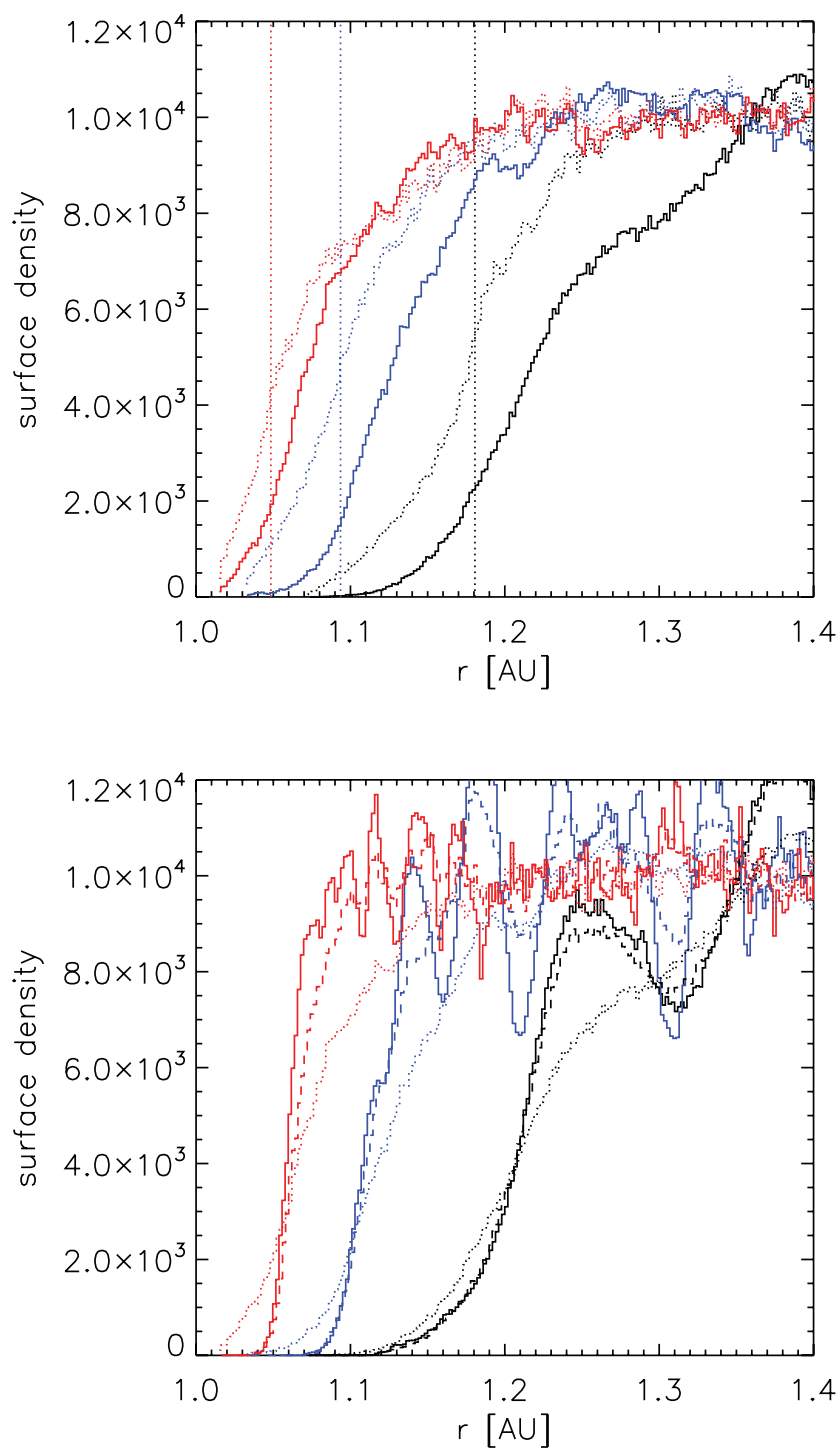

Figure 4. Surface densities at the inner edges of debris discs cleared by a planet at $1 \mathrm{au}$. Top: comparison of the encounter map results (solid) with the prescription of Wisdom (1980; dotted). Vertical dotted lines show the location of the classical zone edge in semimajor axis; irregular dotted lines show the planetesimal distribution when their eccentricities are accounted for. Maximum particle eccentricities are 0.1. Planet masses are $\mu=10^{-3}$ (black), $\mu=10^{-4}$ (blue) and $\mu=10^{-5}$ (red). Bottom: disc profiles according to the encounter map results for different planet masses and planetesimal eccentricities. Planet masses are as above; maximum particle eccentricities are 0.01 (solid), 0.03 (dashed) and 0.1 (dotted). solid lines. We see that the shape of the inner disc edge depends on both the planet's mass, with more massive planets making the edge shallower. We show on the same plot with dotted lines the results from the original prescription of Wisdom (1980), showing a discrepancy both in the location of the edge and in the shape of it. The discrepancy is greater for larger planet mass. In the bottom panel of Fig. 4, we show the results from the encounter map iterations for a range of planet masses and particle eccentricities. More massive planets and more eccentric particles lead to shallower slopes. In addition, fluctuations in the surface density outside the cleared zone caused by mean motion resonances can be seen (see also Quillen 2007), and these are more apparent for lower eccentricities. The shallowness of the edge means that its precise location is ill defined, but if it is attempted to be quantified by a single statistic, for example the half-maximum, then it should be noted that this value depends on the particles' eccentricities, leading to a degeneracy between planet mass and particle eccentricity.

In this subsection, and the subsequent, we consider only the gravitational influence of the planet on the planetesimals which the disc comprises. It is likely that in many debris discs the eccentricities of planetesimals are high, if they have been dynamically excited by planets' secular perturbations (Mustill \& Wyatt 2009) or by large planetary embryos embedded in the disc (Kenyon \& Bromley 2010). In such a disc, collisions between planetesimals will be destructive due to the high relative velocities. Furthermore, eccentricities will remain high as ongoing stirring processes will dominate over collisional damping (Kenyon \& Bromley 2004; Shannon \& Wu 2011). We do note that there may be discs where the relative velocities remain low (Heng \& Tremaine 2010) in which eccentricities may be continually damped by collisions. The truncation of such discs will be governed by both dynamical clearing from the classical chaotic zone and viscous spreading of the disc due to the inelastic collisions (Quillen 2007). We do not consider such discs here.

\subsection{Case study: HR 8799}

As an example of the effects of the extended chaotic zone, consider the HR 8799 system. The $1.5 \mathrm{M}_{\odot}$ star is orbited by four known planets and at least two debris belts. Su et al. (2009) estimated that the inner edge of the outer disc lies at 90 au based on fitting the disc's spectral energy distribution (SED); the inner edge of the disc is not resolved. It is thought that the outermost planet, HR $8799 \mathrm{~b}$, at a projected distance of 68 au from the star (Marois et al. 2008), is responsible for the truncation. Su et al. (2009) argued that the radius of the truncation, the location of planet $\mathrm{b}$ at $68 \mathrm{au}$, and a planet mass of 10 Jovian masses, at the upper end of the range suggested by photometry (Marois et al. 2008), are all consistent with a scenario in which the truncation is due to planet b's chaotic zone. Quantitatively, Su et al. (2009) took the chaotic zone to be given by $1.4 \mu^{2 / 7}$ as given by Malhotra (1998).

As we have seen, this relationship underestimates the width of the chaotic zone at high eccentricities. If the disc particles' eccentricities are above $\sim 0.02$ (estimated from equation 11 ), the extent of the clearing will be greater than this because of the extended chaotic zone. Hence, if the disc particles have low eccentricities, the mass estimated by Su et al. (2009) will be correct, and the planet mass will be around 10 Jovian masses. However, if the disc particles' eccentricities are higher than $\sim 0.02$, the same clearing will be achieved with a smaller planet mass. We quantify this effect in Fig. 5. This compares the shape and extent of the clearing due to planets of 2, 4, 6, 8 and 10 Jovian masses, under the assumptions that particles are removed only in the classical chaotic zone (dotted 


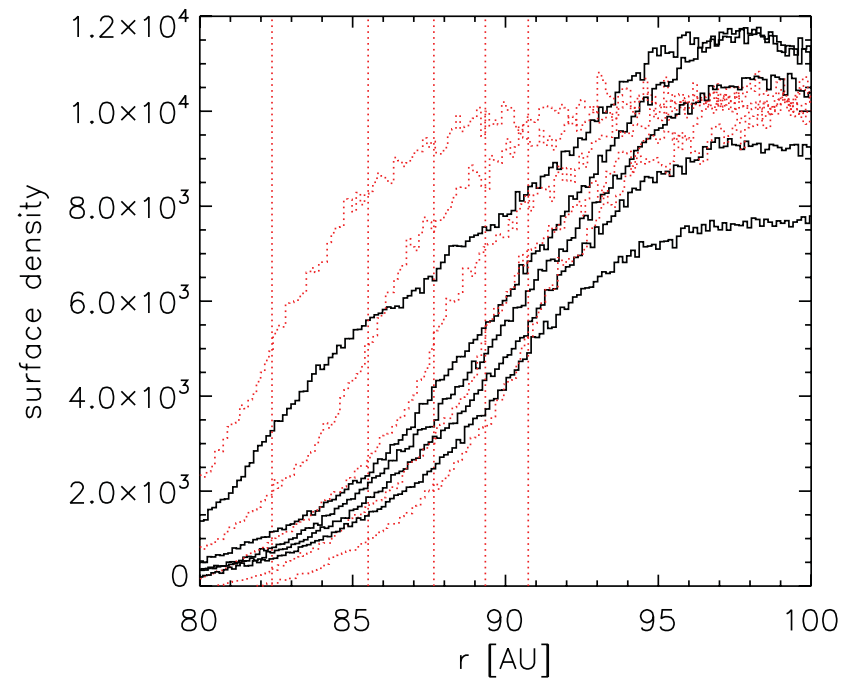

Figure 5. Comparison of disc edge profiles for the disc around HR 8799 truncated by planet $b$ at 68 au. The maximum eccentricity of the disc particles is 0.1. Solid lines show disc profiles derived from the encounter map results; dotted show profiles when truncated by the classical Wisdom prescription. The vertical dotted lines show the location of the disc edge for particles on perfectly circular orbits. Planet masses increase from left to right: $2,4,6$, 8 and 10 Jovian masses. Note that all the disc surface densities are $10^{4}$ far from the planet; the different heights visible here are due to the effects of the 2:1 mean motion resonance at 108 au.

lines) or also in the extended chaotic zone (solid line). In each case, particle eccentricities were distributed uniformly between 0 and 0.1 . We also show as vertical dotted lines the location of the edge for particles on circular orbits. We see that the profiles for 8 and 10 Jovian mass planets under the Wisdom prescription (whose medians straddle $90 \mathrm{au}$ ) are similar to the profiles for the 4 and 8 Jovian mass planets, respectively, under the extended zone prescription. Hence, the planet masses required to achieve a given clearing may be less than predicted by the Wisdom criterion by as much as 50 per cent.

Thus, if the edge of the outer disc is located at $90 \mathrm{au}$, and the particles in the disc have eccentricities of the order of 0.1 , then the mass of planet $b$ is in the range 4-8 Jovian masses, rather than the 810 Jovian masses that it would be if the particles' eccentricities were below 0.02 . For now it is not possible to make such firm conclusions, since observations have yet to resolve the inner edge of the disc, and its $90 \mathrm{au}$ location inferred from SED modelling is degenerate with the assumptions made about the particle properties. Nevertheless, the inner edge will likely be resolved by future observations, and this will help to reduce the uncertainty in the planet mass. For example, some authors, e.g. Currie et al. (2011), claim a lower mass for planet $b$ of 6-7 Jovian masses, and this is consistent with the planet truncating a disc of planetesimals, whose eccentricities range up to 0.1 , at $90 \mathrm{au}$, if the edge indeed be located there. The eccentricities of the disc particles may have been excited to such an extent by the growth of large planetesimals in the disc (Kenyon \& Bromley 2010), secular perturbations from the planets, if one or more is eccentric (Mustill \& Wyatt 2009), or sweeping by mean motion or secular resonances during past evolution of the system (Minton \& Malhotra 2011; Mustill \& Wyatt 2011).

Finally, we note that our main conclusion - that the mass of planet b estimated from the extent of the chaotic zone will depend on the eccentricities of the disc particles - does not depend on the actual location of the disc edge. If the disc prove to be at a greater radius, the mass estimated for a disc of eccentric particles will still be greater than that estimated for particles on circular orbits, although each will be greater than its corresponding mass estimated for a disc edge at $90 \mathrm{au}$.

\subsection{Post-main-sequence evolution of planetary systems}

There are also important implications for the evolution of planetary systems when a star loses mass during post-main-sequence evolution. When stars lose mass on the asymptotic giant branch, the planet:star mass ratios increase and previously stable systems can be destabilized (Debes \& Sigurdsson 2002; Bonsor et al. 2011). Stars typically lose mass at relatively modest rates, and under these conditions orbits expand adiabatically and the ratios of semimajor axes are unchanged (Veras et al. 2011). However, since the planet:star mass ratio increases as the star loses mass, quantities which depend on this ratio, such as the Hill radius and the widths of resonances and the chaotic zone, will change too. The chaotic zone expands, and after mass loss particles which were previously on stable orbits may find themselves in the chaotic zone. The presence of this reservoir of newly unstable material may explain the existence of metal pollution in the atmospheres of white dwarfs (Zuckerman et al. 2003), as well as hot discs orbiting some white dwarfs (Farihi et al. 2009), as bodies are scattered on to highly eccentric orbits and tidally disrupted (Zuckerman et al. 2003; Bonsor et al. 2011). Bonsor et al. (2011) estimated the amount of destabilized material simply by expanding the $\mu^{2 / 7}$ zone according to the new, lower, stellar mass. While this will correctly describe the new chaotic zone at low eccentricity, seemingly vulnerable particles with higher eccentricity may escape being engulfed by the new chaotic zone. This is because the lower boundary of the extended chaotic zone increases as $\mu$ increases. However, a further complication may counteract this: Veras et al. (2011) showed that if mass-loss rates are relatively rapid then particles' orbits do not expand adiabatically and they may acquire some eccentricity. This could push seemingly safe bodies into the extended chaotic zone. Since the eccentricities required to enter the extended chaotic zone are relatively modest, this could be an important mechanism for destabilizing bodies.

\section{ACKNOWLEDGMENTS}

AJM is grateful for the support of an STFC studentship. We are indebted to Amy Bonsor for comments on the manuscript. We should like to thank the anonymous referee for suggesting improvements to the paper.

\section{REFERENCES}

Bonsor A., Mustill A. J., Wyatt M. C., 2011, MNRAS, 414, 930

Chambers J. E., 1999, MNRAS, 304, 793

Chiang E., Kite E., Kalas P., Graham J. R., Clampin M., 2009, ApJ, 693, 734

Chirikov B. V., 1979, Phys. Rep., 52, 263

Currie T. et al., 2011, ApJ, 729, 128

Debes J. H., Sigurdsson S., 2002, ApJ, 572, 556

Duncan M., Quinn T., Tremaine S., 1989, Icarus, 82, 402

Farihi J., Jura M., Zuckerman B., 2009, ApJ, 694, 805

Heng K., Tremaine S., 2010, MNRAS, 401, 867

Henon M., Petit J., 1986, Celest. Mech., 38, 67

Kalas P. et al., 2008, Sci, 322, 1345

Kenyon S. J., Bromley B. C., 2004, AJ, 127, 513

Kenyon S. J., Bromley B. C., 2010, ApJS, 188, 242 
Malhotra R., 1998, in Lazzaro D., Vieira Martins R., Ferraz-Mello S., Fernandez J., eds, ASP Conf. Ser. Vol. 149, Solar System Formation and Evolution. Astron. Soc. Pac., San Francisco, p. 37

Marois C., Macintosh B., Barman T., Zuckerman B., Song I., Patience J., Lafrenière D., Doyon R., 2008, Sci, 322, 1348

Minton D. A., Malhotra R., 2011, ApJ, 732, 53

Moerchen M. M., Churcher L. J., Telesco C. M., Wyatt M., Fisher R. S., Packham C., 2011, A\&A, 526, A34

Murray C. D., Dermott S. F., 1999, Solar System Dynamics. Cambridge Univ. Press, Cambridge

Mustill A. J., Wyatt M. C., 2009, MNRAS, 399, 1403

Mustill A. J., Wyatt M. C., 2011, MNRAS, 413, 554

Quillen A. C., 2006a, MNRAS, 372, L14
Quillen A. C., 2006b, MNRAS, 365, 1367

Quillen A. C., 2007, MNRAS, 377, 1287

Quillen A. C., Faber P., 2006, MNRAS, 373, 1245

Shannon A., Wu Y., 2011, ApJ, 739, 36

Su K. Y. L. et al., 2009, ApJ, 705, 314

Veras D., Wyatt M. C., Mustill A. J., Bonsor A., Eldridge J. J., 2011, MNRAS, 417, 2104

Wisdom J., 1980, AJ, 85, 1122

Zhou L.-Y., Dvorak R., Sun Y.-S., 2011, MNRAS, 410, 1849

Zuckerman B., Koester D., Reid I. N., Hünsch M., 2003, ApJ, 596, 477

This paper has been typeset from a $\mathrm{T}_{\mathrm{E}} \mathrm{X} / \mathrm{LAT}_{\mathrm{E}} \mathrm{X}$ file prepared by the author. 